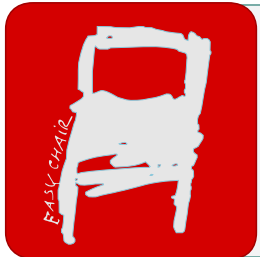

EPiC Series in Health Sciences

CAOS 2018. The 18th Annual Meeting of the International Society for Computer Assisted Orthopaedic Surgery

\title{
"Standard” Versus “Challenging” Patients - Early Outcomes using Computer-Assisted Total Knee Arthroplasty
}

\author{
Yifei Dai ${ }^{1 *}$, Michael P Bolognesi ${ }^{2}$, Samuel S Wellman², Thorsten Seyler ${ }^{2}$, \\ Quanjun Cui ${ }^{3}$, Yassaman Najmabadi ${ }^{1}$, Charlotte Bolch ${ }^{1}$, David Liu ${ }^{4 *}$ \\ ${ }^{1 *}$ Exactech Inc, Gainesville, FL 32653, USA \\ ${ }^{2}$ Duke Orthopaedics, Durham, NC, USA \\ ${ }^{3}$ University of Virginia School of Medicine, Charlottesville, VA, USA \\ ${ }^{4}$ The Gold Coast Centre for Bone and Joint Surgery, Tugun, Queensland, AU \\ yifei.dai@exac.com, dliu01@bigpond.com
}

\begin{abstract}
This study investigated if CAOS TKA cases complicated by challenging patient conditions would negatively impact the perioperative outcomes of surgery. Early outcome data on 51 TKA's from a multicenter, consecutive series were analyzed. The patients were separated into challenging and standard case groups according to the criteria of age, BMI, and severity of deformity. The two groups did not exhibit significant differences in any of the early outcomes. Our study demonstrates consistent early results using CAOS TKA irrespective of patient conditions.
\end{abstract}

\section{Introduction}

Total knee arthroplasty (TKA) is a largely successful surgical treatment for end-stage osteoarthritis. However, it has been shown that some challenging conditions, such as advanced age, obesity, and severe deformity or stiffness can increase surgical time and length of stay, as well as the risk of inferior function and early failure for conventional TKA [1-4]. These findings indicate that the type of patient or local condition of the knee may sometimes challenge the results of the surgery.

Computer-assisted orthopedic system (CAOS) TKA has been introduced as an advanced surgical tool intended to provide intraoperative guidance that may mitigate the impact of these challenges, potentially making the surgery particularly beneficial for the "challenging" case. Multiple studies on CAOS TKA concluded that it offers significant advantages in both intraoperative outcomes and implant alignment [5-7]. However, limited data is available assessing the outcomes of challenging patient 
"Standard" Versus "Challenging" Patients - Early Outcomes using Computer-Assisted ... Y. Dai et al.

conditions as compared to "standard" TKA cases. Current available reports on challenging CAOS TKA outcomes have mainly focused on the impact of coronal extra-articular deformity [8-10].

The goal of this study was to investigate if CAOS TKA also exhibits differences between challenging and standard cases in terms of the early outcomes, similar to those observed with conventional TKA.

\section{Materials and Methods}

With institutional review board-approval and patient's signed informed consent, a multicentre, consecutive case series study was conducted by 5 surgeons in a total of 51 patients (51 knees). All cases were primary TKA using the Optetrak Logic ${ }^{\circledR}$ Knee System (Exactech Inc, Gainesville, FL, USA) with the assistance of a contemporary CAOS system (ExactechGPS ${ }^{\circledR}$, Blue-Ortho, Gieres, FR).

"Challenging" cases were identified from the series with one or more of the following conditions: 1) age greater than 80 years, 2) BMI greater than 35,3 ) coronal deformity greater than $15^{\circ}$, and 4 ) range of flexion (ROM) less than $90^{\circ}$. The remaining cases were grouped as "standard" cases. Early outcomes from the 6-month postoperative visit were compared between the standard and challenging case groups. Patients were assessed for: Range of Motion (ROM), the Hospital for Special Surgery Knee Score (HSS), the 2011 Knee Society Score (KSS), and the Knee Injury and Osteoarthritis Outcome Score (KOOS). In addition, patient satisfaction in Visual Analog Scale (VAS, 1 to 10 with 10 indicates highest satisfaction) at 6-month postoperatively visit and implant related complications were reviewed. Statistical significance was defined as $\mathrm{p}<0.05$.

\section{Results}

Nineteen patients were identified as challenging cases, whereas the remaining thirty-two patients were grouped as standard cases (Table 1). A summary of 6-month outcomes in the standard and challenging groups is presented in Table 2 . The challenging patients were found to have approximately $9^{\circ}$ less flexion compared to the standard patients. A general trend was observed that the challenging cases tended to gain more improvement in outcome scores (Table 2), though the differences in improvement were statistically insignificant between the two groups. All the gains in clinical scores in both groups were well above the Minimal Clinically Important Difference (MCID), suggesting significant improvement in the condition of these patients in both groups after TKA. Similarly, no statistical difference was found in the 6-month outcome scores and patient satisfaction VAS between the groups. No implant related early complications were reported in this series. 
"Standard" Versus "Challenging" Patients - Early Outcomes using Computer-Assisted ... Y. Dai et al.

\begin{tabular}{lccc}
\hline & Difficult TKA & Standard TKA & P \\
N & 19 & 32 & - \\
Age (years) & $63.5 \pm 10.4$ & $65.6 \pm 9.2$ & 0.486 \\
Female (\%) & 68 & 44 & 0.001 \\
BMI & $36.0 \pm 6.7$ & $29.8 \pm 2.7$ & 0.001 \\
Count of Comorbidities* & $2.7 \pm 1.0$ & $2.6 \pm 0.8$ & 0.603 \\
${ }^{*}$ The total number of comobidities per patient. & & & \\
ROM $\left(^{\circ}\right)$ & $100.8 \pm 18.6$ & $112.4 \pm 8.4$ & 0.017 \\
HSS & $56.7 \pm 16.2$ & $64.4 \pm 10.0$ & 0.076 \\
OKS & $20.1 \pm 9.6$ & $22.4 \pm 7.1$ & 0.363 \\
KSS & & & \\
Function & & & 0.277 \\
Knee & $46.3 \pm 18.8$ & $52.3 \pm 18.9$ & 0.017 \\
KOOS & $48.0 \pm 12.4$ & $57.2 \pm 13.3$ & \\
$\quad$ Pain & & & 0.473 \\
Symptoms & $41.5 \pm 19.6$ & $45.4 \pm 16.0$ & 0.676 \\
ADL & $41.7 \pm 21.8$ & $44.1 \pm 14.5$ & 0.332 \\
QOL & $45.6 \pm 21.0$ & $50.9 \pm 13.3$ & 0.550 \\
\hline
\end{tabular}

Table 1. Baseline patient characteristics of the challenging and standard case groups.

\begin{tabular}{|c|c|c|c|c|}
\hline & Challenging Patients & Standard Patients & $\mathrm{P}$ & MCID [Reference] \\
\hline $\operatorname{ROM}\left({ }^{\circ}\right)$ & $109.5 \pm 13.4$ & $118.1 \pm 13.0$ & 0.032 & \\
\hline Satisfaction VAS (1-10) & $8.9 \pm 2.3$ & $8.6 \pm 2.4$ & 0.603 & \\
\hline \multicolumn{5}{|c|}{ Improvement in Outcomes from Baseline to $6 \mathrm{Mo}$} \\
\hline HSS & $19.6 \pm 21.1$ & $16.5 \pm 13.7$ & 0.597 & $8.29[12]$ \\
\hline OKS & $15.7 \pm 10.3$ & $12.3 \pm 10.2$ & 0.250 & $3-5[13]$ \\
\hline KSS & $44.8 \pm 42.2$ & $38.5 \pm 42.7$ & 0.635 & $34.5[14]$ \\
\hline Function & $23.7 \pm 24.4$ & $18.8 \pm 24.5$ & 0.490 & \\
\hline Knee & $24.4 \pm 22.2$ & $21.1 \pm 23.3$ & 0.640 & \\
\hline \multicolumn{5}{|l|}{ KOOS } \\
\hline Pain & $35.5 \pm 22.2$ & $29.7 \pm 24.2$ & 0.414 & \multirow{4}{*}{$\begin{array}{l}8 \mathrm{pts} \text { in each } \\
\text { subscale } \\
\text { scores [15] }\end{array}$} \\
\hline Symptoms & $26.7 \pm 24.2$ & $25.3 \pm 22.4$ & 0.832 & \\
\hline ADL & $30.8 \pm 26.5$ & $27.5 \pm 22.3$ & 0.649 & \\
\hline $\mathrm{QOL}$ & $38.7 \pm 31.4$ & $34.0 \pm 29.6$ & 0.600 & \\
\hline
\end{tabular}

Table 2. Early outcomes at 6-months postoperatively.

\section{Discussion}

This study demonstrates that contrary to conventional TKA, CAOS TKA patients challenged by conditions such as BMI, age, and deformity did not exhibit inferior outcomes in response to the surgery except for ROM, compared to the standard cases. Even with the slight less ROM in the early outcome of challenging patients compared to standard patients, both group achieved on average $\geq 110^{\circ}$ flexion, which is sufficient for most daily activities. It should be noted this study only investigated the impact 
"Standard" Versus "Challenging" Patients - Early Outcomes using Computer-Assisted ... Y. Dai et al.

of challenging conditions in early TKA outcomes (6-month postop). Longer-term follow-up is needed to determine the stabilized clinical outcomes in the future study.

As CAOS TKA has been proven to offer significant improvement in surgical accuracy compared to conventional TKA, cases with challenging patient conditions are perceived to especially benefit from the intraoperative surgical guidance, thereafter leading to more consistent outcomes compared to standard cases. This benefit has been already demonstrated by case studies and clinical series on patients with severe coronal deformity [5-7]. The findings from this study suggested that CAOS TKA may be considered as an effective and appealing option in the cases with challenging patient conditions.

\section{References}

[1] Teeney SM, Krackow KA, Hungerford DS, Jones M. Primary total knee arthroplasty in patients with severe varus deformity. Clin Orthop Relat Res 1991;273:19-31.

[2] Krushell RJ, Fingeroth RJ. Primary total knee arthroplasty in morbidly obese patients: a 5 to 14 year follow up study. J Arthroplast 2007;22(6 suppl 2):77-80.

[3] Foran JR, Mont MA, Etienne G, Jones LC, Hungerford DS. The outcome of total knee arthroplasty in obese patients. J Bone Jt Surg [Am] 2004;86-A(8):1609-15.

[4] Kuperman EF, Schweizer M, Joy P, Gu X, Fang MM. The effects of advanced age on primary total knee arthroplasty: a meta-analysis and systematic review. BMC Geriatrics. 2016;16:41.

[5] Smith ID, Elton R, Ballantyne JA, Brenkel IJ. Pre-operative predictors of the length of hospital stay in total knee replacement. J Bone Joint Surg Br. 2008;90(11):1435-40.

[6] Brin YS, et al. Imageless computer assisted versus conventional total knee replacement. A Bayesian meta-analysis of 23 comparative studies. Int Orthop,35(3):331-9,2011.

[7] Hetaimish BM, et al. Meta-analysis of navigation vs conventional total knee arthroplasty. J Arthroplasty,27(6):1177-82,2012.

[8] Chauhan SK, Scott RG, Breidahl W, Beaver RJ. Computer-assisted knee arthroplasty versus a conventional jig-based technique. A randomised, prospective trial. J Bone Joint Surg Br. 2004;86(3):372-7.

[9] Mullaji A, Shetty GM. Computer-assisted total knee arthroplasty for arthritis with extra-articular deformity. J Arthroplasty. 2009;24(8):1164-9.

[10] Bottros J, Klika AK, Lee HH, Polousky J, Barsoum WK. The use of navigation in total knee arthroplasty for patients with extra-articular deformity. J Arthroplasty. 2008;23(1):74-8.

[11] Kuo CC, Bosque J, Meehan JP, Jamali AA. Computer-assisted navigation of total knee arthroplasty for osteoarthritis in a patient with severe posttraumatic femoral deformity. J Arthroplasty. 2011;26(6):976.e17-20.

[12] Sigh JA, Schleck C, Harmsen S, et al. Validation of the hospital for special surgery knee questionnaire: convergent validity, responsiveness and sensitivity to change. 2013 ACR/ARHP Annual Meeting.

[13] Murray DW, Fitzpatrick R, Rogers K, Pandit H, Beard DJ, Carr AJ, Dawson J. The use of the Oxford hip and knee scores. J Bone Joint Surg Br. 2007;89(8):1010-4.

[14] C. A. Jacobs and C. P. Christensen, Correlations between knee society function scores and functional force measures. Clinical Orthopaedics and Related Research. 2009;467(9):2414-9.

[15] Roos EM, Lohmander LS. The Knee injury and Osteoarthritis Outcome Score (KOOS): from joint injury to osteoarthritis. Health and Quality of Life Outcomes. 2003;1:64. 